\title{
Thiolation in arsenic metabolism: a chemical perspective
}

\author{
C. Fan ${ }^{1}$, G. Liu ${ }^{1} \&$ Y. Cai ${ }^{1,2}$ \\ ${ }^{1}$ Department of Chemistry and Biochemistry, Florida International University, Miami, FL, USA \\ ${ }^{2}$ Southeast Environmental Research Center, Florida International University, Miami, FL, USA
}

\begin{abstract}
In recent years, methylated thioarsenicals have been widely detected in various biological and environmental matrices, suggesting their broad involvement and biological implications in arsenic metabolism. However, very little is known about the formation mechanisms of methylated thioarsenicals and the relation between arsenic methylation and thiolation processes. It is timely and necessary to summarize and synthesize the reported information on thiolated arsenicals for an improved understanding of arsenic thiolation. To this end, we examined the proposed formation pathways of methylated oxoarsenicals and thioarsenicals from a chemical perspective, and proposed a new arsenic metabolic scheme in which arsenic thiolation is integrated with methylation processes (instead of being separated from methylation as currently reported), followed lastly by discussion on the biological implications of the new scheme of arsenic metabolism. This informative review on arsenic thiolation from the chemical perspective will be helpful to better understand the arsenic metabolism at the molecular level and the toxicological effects of arsenic species.
\end{abstract}

\section{INTRODUCTION}

Arsenic is a toxic metalloid widely distributed in the environment and poses health risk to human beings. On the other hand, arsenic has been recognized in successful treatment of cancers (e.g., multiple myeloma). The double-edged effects of arsenic are highly dependent on the arsenic species and their metabolic processes in living organisms. Arsenic metabolism is a complex process involving a series of biochemical reactions including oxidation, reduction, methylation, and thiolation. Methylation process was considered a major arsenic metabolic pathway, and the end products would be methylated oxyarsenicals (Cullen, 2014; Dheeman et al., 2014). Methylated thioarsenicals, referring to pentavalent species in this review unless stated otherwise and including monomethylmonothioarsinic acid (MMMTA(V)), dimethylmonothioarsinic acid (DMMTA(V)) and dimethyldithioarsinic acid (DMDTA(V)), were recently found as common arsenic metabolites and may be of important biological implications, as they were demonstrated to be more toxic than their oxycounterparts, possibly because of their capability of binding proteins. The arsenic metabolic pathways proposed previously focused predominantly on methylated oxoarsenicals, but very limited studies reported sporadic, inconsistent information on thiolation process independent of arsenic methylation (Sun et al., 2016; Wang et al., 2015). It is urgently needed to review the reported information on the formation of methylated thioarsenicals and more importantly, to use a holistic approach to systematically consider both methylation and thiolation processes during arsenic metabolism.

\section{FORMATION PATHWAYS OF METHYLATED OXOARSENICALS AND THIOARSENICALS}

Previously proposed arsenic methylation pathways including classic oxidative methylation, reductive methylation, and the processes involving proteinbound arsenicals were reviewed from a chemical perspective. We found that the oxidative methylation pathway is more chemically plausible, regardless of the arsenic binding to proteins or not. Pentavalent arsenicals seem to be obligated intermediates or exist in the transition state during the methylation reactions. These proposed arsenic methylation pathways were ended up with forming methylated oxoarsenicals, contributing little to explain the increasingly common detection of thioarsenicals. Arsenic thiolation process was regarded as a separated pathway from methylation process, and methylated thioarsenicals were presumably produced through 1) thiolation of methylated arsenicals (free or protein-bound) and 2) thiolation of iAs following successive methylation. These chemical reactions were studied, and direct thiolation of pentavalent arsenic was found to be more likely to occur.

\section{A NEWLY PROPOSED FORMATION PATHWAY OF METHYLATED THIOARSENICALS}

A new formation pathway of methylated thioarsenicals accompanying methylation process was proposed (Fig. 1). The new arsenic metabolic pathway takes into account thiolation and methylation simultaneously while considering the fact that most arsenic 


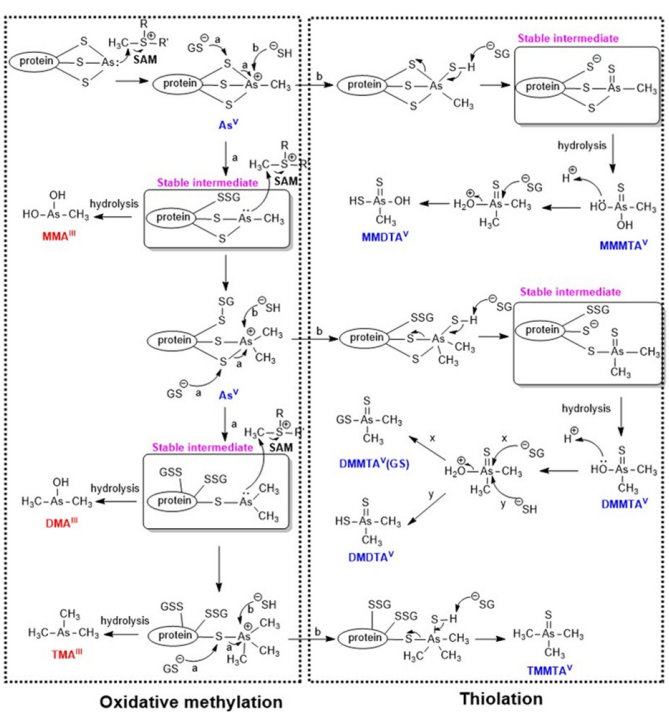

Figure 1. Proposed arsenic metabolic scheme integrating methylation with thiolation.

are bound to proteins in the biological matrix before being excreted. Trivalent inorganic arsenicals undergo an oxidative methylation in the presence of Sadenosylmethionine (SAM) while they are bound to proteins or enzymes. After accepting a methyl group from SAM, the arsenicals are oxidized to pentavalent state while still being bound to proteins. We proposed that the resulting intermediates could undergo reduction (process a) or thiolation (process b) to form stable protein-bound products. The reduction process yields trivalent protein-bound arsenicals in the presence of reductive reagents, such as glutathione $(\mathrm{GSH})$ (process a). The thiolation process could be a competitive reaction to form stable pentavalent protein-bound arsenicals to keep their oxidative state (process b). Reduction and thiolation processes produce protein-bound methylated trivalent arsenicals and pentavalent thioarsenicals, respectively. These two types of protein-bound arsenicals are stable and crucial intermediates in arsenic metabolism.

Although lack of techniques to directly detect the protein-bound arsenic species, the evidence from previous studies related to the formation of the proposed intermediates was reviewed in this article. For example, DMMTA ${ }^{\mathrm{V}}$ was demonstrated to form a stable As-S bond with GSH and the product, DMMTA(V)(GS), as a stable arsenic metabolite, was found in Brassica oleracea extracts after exposure to dimethylarsinic acid $(\mathrm{DMA}(\mathrm{V}))$ as well as in human cancer cell line treated with darinaparsin (Raab et al., 2007; Yehiayan et al., 2014).

\section{IMPLICATIONS OF THE PROPOSED MECHANISM}

According to the new pathway, methylated thioarsenicals can be produced through reaction of sulfide with not only free arsenic metabolites but also protein-bound arsenic intermediates. Thiolation reaction occurs competitively with methylation reaction, and methylated thioarsenicals should be important metabolites rather than occasional by-products. After uptake, most arsenic species are bound to proteins in biological systems. The protein-bound arsenicals used to be considered as only in the trivalent forms. Pentavalent thioarsenicals also can bound to proteins to form stable complexes. Therefore, free thioarsenicals, just like free trivalent arsenicals, should be usually found in low concentrations and could be easily ignored. Thioarsenicals can convert to oxyarsenicals during sampling, storage, preparation, and analysis, which could cause underestimation of the amount of thioarsenicals. Methylation of inorganic arsenic was previously regarded as a detoxification pathway due to the formation of less toxic monomethylarsonic acid $(\mathrm{MMA}(\mathrm{V}))$ and $\mathrm{DMA}(\mathrm{V})$, until their trivalent forms, monomethylarsonous acid (MMA(III)) and dimethylarsinous acid (DMA(III)), were detected. Similarly, pentavalent arsenic was considered to be less toxic because of low binding affinity to proteins, however, $\operatorname{MMMTA}(\mathrm{V})$ and DMMTA(V) were demonstrated to be highly toxic. The high toxicity, on the other hand, could make thioarsenicals potential anticancer drug candidates. The newly proposed arsenic metabolic pathway could provide valuable information for study of arsenic-based anticancer medicines.

\section{REFERENCES}

Cullen, W.R. 2014. Chemical mechanism of arsenic biomethylation. Chem. Res. Toxicol. 27(4): 457-461.

Dheeman, D.S., Packianathan, C., Pillai, J.K. \& Rosen, B.P. 2014. Pathway of human AS3MT arsenic methylation. Chem. Res. Toxicol. 27(11): 1979-1989.

Raab, A., Wright, S.H., Jaspars, M., Meharg, A.A. \& Feldmann, J. 2007. Pentavalent arsenic can bind to biomolecules. Angew. Chem. 119: 2648-2651.

Sun, Y., Liu, G. \& Cai, Y. 2016. Thiolated arsenicals in arsenic metabolism: occurrence, formation, and biological implications. J. Environ. Sci. 49: 59-73.

Wang, Q.Q., Thomas, D.J. \& Naranmandura, H. 2015. Importance of being thiomethylated: formation, fate, and effects of methylated thioarsenicals. Chem. Res. Toxicol. 28(3): 281-289.

Yehiayan, L., Stice, S., Liu, G., Matulis, S., Boise, L.H. \&Cai, Y. 2014. Dimethylarsinothioyl glutathione as a metabolite in human multiple myeloma cell lines upon exposure to darinaparsin. Chem. Res. Toxicol. 27(5): 754-764. 